\title{
ROTEIRIZAÇÃO DE NAVIOS COM CONTROLE DE ESTOQUE NA INDÚSTRIA PETROLÍFERA: CONTRIBUIÇÕES EM MODELAGEM MATEMÁTICA E PROPOSTA DE UMA HEURÍSTICA DE BUSCA LOCAL
}

\author{
Amélia Stanzani \\ Departamento de Engenharia de Produção - UFSCar - São Carlos-SP \\ alstanzani@gmail.com \\ Vitória Pureza \\ Departamento de Engenharia de Produção - UFSCar - São Carlos-SP \\ vpureza@dep.ufscar.br \\ Reinaldo Morabito \\ Departamento de Engenharia de Produção - UFSCar - São Carlos-SP \\ morabito@dep.ufscar.br
}

\begin{abstract}
Resumo
O presente artigo discute um problema real de roteirização de navios e controle de estoque nos pontos de suprimento (plataformas offshore) e atendimento de demanda nos pontos consumidores (terminais costeiros) presente nas operações de coleta e entrega de diferentes tipos de óleo cru de empresas petrolíferas. O transporte dos produtos é em grande parte o resultado da necessidade de manutenção dos estoques em cada plataforma entre níveis mínimos e máximos. A roteirização e programação da frota visa a obtenção de soluções de mínimo custo variável, e deve considerar diversas restrições operacionais, dentre as quais destaca-se a viabilidade de atracação de navios em portos dos pontos operacionais e descarregamentos simultâneos em terminais com mais de um berço. Neste trabalho, exemplares com dados reais obtidos a partir de um estudo de caso em uma empresa petrolífera nacional, foram resolvidos a partir da implementação de um modelo linear inteiro misto que descreve o problema e por um método heurístico de busca local.

Palavras-chave: Problema de roteirização e controle de estoque, transporte marítimo de petróleo, limitação de berços, heurística de busca local.
\end{abstract}

\begin{abstract}
This paper discusses a real ship routing problem with inventory control in supply points (offshore platforms) and demand requirements in consumers points (coastal terminals) which appear in pickup and delivery operations of different types of crude oil. The transportation of the products is largely the result of the need to maintain the inventory in each platform between minimum and maximum levels. The routing and scheduling of the fleet aims to obtain minimum variable cost solutions, and should consider several operational constraints, such as the feasibility of docking the vessels at the ports and simultaneous dockings in terminals with more than one berth. In this work, real data examples from a case study in a national oil company, were solved by means of an implementation of a mixed integer linear model that describes the problem and by a local search heuristic.
\end{abstract}

Keywords: Inventory routing problem, shipping oil, limitations of berths, local search heuristic. 


\section{INTRODUÇÃO}

O problema considerado neste artigo trata das decisões logísticas envolvidas na coleta e entrega de óleo cru realizadas por empresas petrolíferas entre plataformas de prospecção offshore (pontos de suprimento) e terminais costeiros (pontos de demanda). $\mathrm{O}$ transporte desses produtos na empresa estudada é realizado por uma frota de navios heterogênea, sendo em grande parte acionado pela necessidade da não interrupção da produção nas plataformas e do atendimento da demanda nos terminais. Soluções para o problema visam a coordenação da gestão do estoque e do transporte, buscando determinar simultaneamente estoques ótimos e uma estratégia de distribuição que minimizem o custo da operação. Tal característica define o problema como um problema integrado de Roteirização de Veículos e Controle de Estoque (IRP - Inventory Routing Problem).

Assim como a maioria dos problemas de roteirização de interesse, o IRP tem natureza combinatória, e é considerado de difícil resolução (NP-difícil), o que faz seu tratamento um desafio para profissionais da prática e pesquisadores. Uma das motivações para esse objeto de estudo é a expressiva relevância prática de problemas de roteirização de navios, uma vez que aproximadamente $90 \%$ do volume e $70 \%$ do valor total das mercadorias transportadas em todo o mundo são levadas pelo mar (Hwang, 2005).

Christiansen et al. (2013) apresentam uma extensa revisão bibliográfica de problemas de roteirização e programação de navios impulsionados pela quantidade expressiva de trabalhos relacionados a esse tema publicados na última década, alguns dos quais discutidos na Seção 2. Nesse período, o transporte marítimo internacional, relacionado principalmente a empresas petrolíferas e químicas, teve uma expansão de $40 \%$ e a busca pela eficiência na operação de transporte nesse modal é fator motivador de pesquisas nessa área de estudo.

\section{PROBLEMAS DE ROTEIRIZAÇÃO E CONTROLE DE ESTOQUE NO CONTEXTO MARÍTIMO}

O IRP marítimo foi primeiramente discutido na pesquisa de Miller (1987), abrindo caminho para pesquisas neste tema. Christiansen e Nygreen (1998a e 1998b) discutiram o IRP marítimo mono-produto com restrições de janela de tempo tanto nos pontos de coleta como de entrega. As quantidades carregadas e descarregadas são determinadas pelas taxas de produção das unidades, manutenção dos níveis de estoque, e a capacidade dos navios que visitam os portos de carregamento/descarregamento. A situação estudada é formulada como um problema de programação linear inteira mista, aplicado posteriormente a um problema real de transporte de amônia de uma empresa norueguesa entre pontos de produção e de consumo em Christiansen (1999). A formulação matemática do problema real abordado pelos autores, apresenta restrições básicas de fluxo em rede, conservação de carga, fluxo temporal, controle de estoque, dentre outras, para o caso monoproduto. Os resultados com uma abordagem de decomposição de Dantzig-Wolfe revelaram ser esta uma boa ferramenta para auxiliar a empresa no planejamento regional das operações de coleta e entrega.

Aplicações do IRP marítimo no transporte de múltiplos produtos representam um desafio para pesquisas futuras, principalmente relacionadas a controle de estoque de cada produto e possível incompatibilidade de produtos em um mesmo compartimento do navio. Nesse sentido, várias pesquisas recentes foram desenvolvidas, especialmente voltadas para indústrias químicas e petrolíferas, das quais cabe citar os trabalhos de Fagerholt (1999), Al-Khayyal e Hwang (2007) e Li et al. (2010), em que se considera que os navios têm compartimentos dedicados para produtos específicos. O IRP marítimo com múltiplos produtos é também tratado nos trabalhos de Persson e Göthe-Lundgren (2005), Christiansen et al. (2011), Siswanto et al. (2011), Agra (2013) e Agra (2014) onde são considerados compartimentos não dedicados.

Em Al-Khayyal e Hwang (2007), o modelo de Christiansen (1999) é estendido para o caso de múltiplos produtos com carregamento/descarregamento simultâneo de múltiplos navios no mesmo porto. Devido à natureza dos produtos, não se permite transportar mais de dois deles no mesmo compartimento. Como estratégia de solução do modelo foi utilizada a relaxação 
lagrangeana para obter limitantes inferiores do valor ótimo. O problema pode ser decomposto em vários subproblemas mais tratáveis; um conjunto de subproblemas é resolvido utilizando a técnicas de fluxo de rede, enquanto outro conjunto de subproblemas é resolvido por programação inteira com um número de variáveis binárias relativamente pequeno. Para obter uma solução viável para exemplares de maior porte os autores também propõem dois algoritmos heurísticos construtivos.

O desenvolvimento de métodos heurísticos para tratar problemas de roteirização e controle de estoque de grande porte estão presentes em grande parte dos trabalhos nessa temática, uma vez que incorporam características específicas do problema estudado em cada caso, produzindo soluções de qualidade em um tempo de processamento pequeno, e podem servir determinar boas soluções iniciais para modelos matemáticos e algoritmos exatos. É o caso dos trabalhos desenvolvidos por Stalhane et al. (2012), Al-Khayyal e Hwang (2007), entre outros.

A consideração do número de berços fornece um elemento mais realista na descrição de várias aplicações, como a aplicação objeto deste artigo. Em seu trabalho, Halvorsen-Weare e Fagerholt (2010) discutem a um estudo de caso de uma empresa de gás natural liquefeito em que o número de atracações simultâneas (correspondentes ao número de berços) por período é incorporado ao modelo matemático com horizonte de planejamento discretizado, por sua vez, resolvido por um método de decomposição das decisões de programação e roteirização.

\section{O PROBLEMA DE COLETA E ENTREGA DE ÓLEO CRU DE PLATAFORMAS OFFSHORE A TERMINAIS COSTEIROS}

\subsection{DESCRIÇÃO DO PROBLEMA}

O transporte dos produtos de plataformas offshore a terminais costeiros da empresa estudada tem como objetivo a minimização do consumo de combustível dos navios considerados no horizonte de planejamento. As rotas devem ser elaboradas respeitando um conjunto de restrições operacionais específicas da aplicação. As plataformas produzem continuamente um único produto a uma taxa constante de produção, e permitem a atracação de apenas um navio por vez. Os terminais demandam um ou mais produtos e é permitido o descarregamento simultâneo de dois ou mais navios, limitado ao número máximo de berços de cada porto.

Os berços dos terminais possuem limitações físicas que podem impedir a atracação de navios considerando suas dimensões (Figura 1). Se o comprimento do navio (length overall LOA) for maior que o comprimento máximo que um determinado berço de um terminal comporta, a atracação é incompatível. O berço de um terminal também impede a atracação de navios cujo calado (distância vertical da parte inferior do navio e a superfície da água) seja maior que a profundidade do berço. Na prática o calado de um navio depende da carga a bordo, de maneira que em certos terminais é permitida a atracação de navios com calado nominal superior à profundidade dos berços, desde que a capacidade ocupada dos navios seja inferior a uma dada porcentagem.

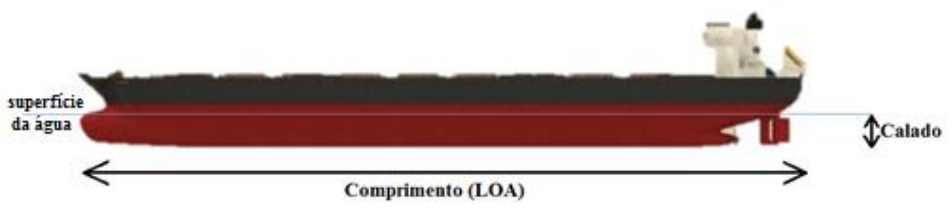

Figura 1 - Dimensões de um navio. Fonte: Adaptado de SOBERANA (2013).

A atracação em plataformas, por outro lado, depende das características da plataforma (FPSO ou fixa) e do navio (com posicionamento dinâmico ou convencional). As plataformas de produção podem ser fixas, montadas sobre uma estrutura de suporte, denominada jaqueta, que se apoia no fundo do mar, ou FPSO (Floating, Production, Storage and Offloading), que são unidades flutuantes de produção, armazenamento e transferência de petróleo, construídas a partir de um navio e fixadas no seu local de produção por um sistema de turret (torre), uma estrutura 
que permite que o navio gire ao redor da mesma sem interromper a produção. A Figura 2 a seguir ilustra esses dois tipos de plataformas.

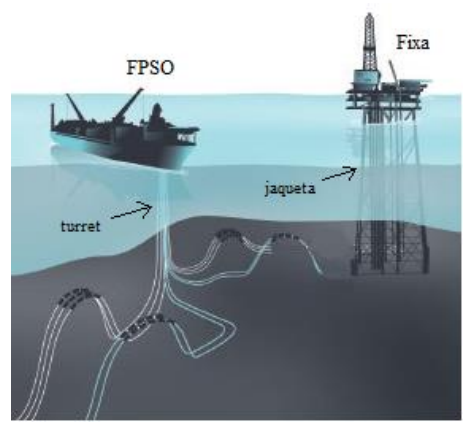

Figura 2 - Tipos de Plataformas.

Fonte: Adaptado de Revista Notícias do Trecho (2014).

Já os navios podem ser convencionais ou equipados com um sistema de posicionamento dinâmico (Dynamic Position - DP). O sistema DP dos navios controla automaticamente a posição e aproamento por meio de propulsão ativa, permitindo posicionamento de navios, em um ângulo favorável em relação à direção dos ventos, correntezas e ondulações, o que permite respostas rápidas às variações climáticas e exigências operacionais.

A Tabela 1 apresenta os requisitos de atracação para cada tipo de navio e plataforma. Note que atracação de um navio convencional em uma plataforma fixa não é permitida devido ao risco de colisão provocado por ondulações e ventos.

Tabela 1 - Porcentagem máxima da capacidade ocupada de um navio para permitir a atracação em uma plataforma.

\begin{tabular}{|c|c|c|}
\hline Plataformas & FPSO & Fixa \\
\hline Posicionamento Dinâmico & $50 \%$ & $50 \%$ \\
\hline Convencional & $30 \%$ & $\begin{array}{c}\text { Incompatibilidade de } \\
\text { Atracação }\end{array}$ \\
\hline
\end{tabular}

A empresa estudada define lotes de produtos com origens (plataformas), destinos (terminais) e datas pré-estabelecidos, considerando indiretamente as taxas de produção das plataformas e a demanda por produtos de suas refinarias. Portanto, a operação real pode ser vista como um problema de Coleta e Entrega (Pick up and Delivery Problem - PDP) com lotes rígidos de produtos com origem e destino conhecidos. Note, entretanto, que a modelagem do problema como um IRP, utilizada no presente artigo, proporciona uma melhor utilização dos recursos, uma vez que as quantidades coletadas são decisão do problema. Como a produção das plataformas é considerada ininterrupta, tratar o problema real como um IRP torna a operação mais próxima da prática da empresa, de que as coletas sejam guiadas pela manutenção do estoque nas plataformas entre os níveis máximo e mínimo.

\subsection{MODELAGEM MATEMÁTICA}

A modelagem matemática é o resultado da adaptação dos modelos para o IRP presentes em Christiansen (1999) e Al-Khayyal e Hwang (2007), considerando características específicas das operações de coleta e entrega da empresa estudada e ausentes nos modelos citados. Em particular, são propostas restrições de limitação no número máximo de descarregamentos simultâneos nos terminais de consumo em um modelo de tempo contínuo, restrições de atracação de navios em plataformas, relacionadas à presença de posicionamento dinâmico nos navios e ao tipo de plataforma, e restrições de atracação em terminais relacionadas ao calado máximo dos navios. A notação utilizada e o modelo matemático são descritos a seguir. 


\section{Índices:}

$i, j$ : pontos operacionais (POs)(terminais e plataformas);

$v, w$ : navios;

$i_{v}$ : POs de posição inicial de navios;

$m, n, \tau$ : visitas aos POs;

$p$ : produtos;

$t$ : terminais;

$i_{p}$ : plataforma de produção do produto $p$.

\section{Conjuntos:}

$H$ : conjunto total de POs;

Hp: conjunto de plataformas;

$H t$ : conjunto de terminais;

$V$ : conjunto total de veículos;

$H_{v}$ : conjunto de POs que podem ser servidos pelo navio $v$, inclusive nós iniciais de navios $(v \in V)$;

$P F$ : conjunto de plataformas fixas;

$V D P$ : conjunto de navios com posicionamento dinâmico;

$M_{i}$ : conjunto das visitas ao PO $i$;

$P T$ : conjunto total de produtos;

$P_{i}$ : conjunto de produtos produzidos ou demandados pelo PO $i$.

\section{Dados de entrada:}

$t_{i j}$ : tempo de viagem do PO $i$ ao PO $j$, em horas;

$c a_{i}$ : custo de atracação do PO $i$, em unidades monetárias por visita;

$c m_{v}$ : custo em movimento navio $v$, em unidades monetárias por dia;

$c s b_{v}$ : custo stand by navio $v$, em unidades monetárias por dia;

$l o_{p v}$ : quantidade inicial de produto $p$ a bordo do navio $v, \mathrm{em} \mathrm{m}^{3}$;

$C A P_{v}$ : capacidade do navio $v$, em $\mathrm{m}^{3}$;

$\alpha_{i v}$ : porcentagem máxima da capacidade ocupada do navio $v$ antes de atracar no terminal $i$; $Q M I N_{i m}$ : quantidade mínima de produto a ser carregada ou descarregada no PO $i$ na visita $m, \mathrm{em} \mathrm{m}^{3}$;

liberacao $_{v}$ : instante de liberação do navio $v$ em sua localização inicial, em horas;

$T$ : horizonte de planejamento, em horas;

$T U_{v}$ : tempo de descarregamento de uma unidade do produto do navio $v$ para um terminal qualquer, em horas;

$T L_{i}$ : tempo de carregamento de uma unidade do produto da plataforma $i$ para um navio qualquer, em horas;

$T W S_{i}$ : instante de abertura da janela de tempo no PO $i$, em horas;

$T W E_{i}$ : instante de fechamento da janela de tempo no PO $i$, em horas;

$s o_{i p}$ : nível de estoque inicial de produto $p$ no $\mathrm{PO} i$, em $\mathrm{em}^{3}$;

$R_{i p}$ : taxa de produção ou consumo do produto $p$ no PO $i$, em $\mathrm{m}^{3}$ por hora;

$S M i n_{i p}$ : estoque mínimo de produto $p$ no $\mathrm{PO} i$, em $\mathrm{m}^{3}$;

$S$ Max $_{i p}$ : estoque máximo de produto $p$ no $\mathrm{PO} i, \mathrm{em} \mathrm{m}^{3}$;

$M u_{i}$ : última visita possível ao PO $i$;

$B_{i}$ : quantidade de berços do terminal $i$;

$T S$ : tempo de serviço;

$d_{i e m_{i p}}$ quantidade total demanda de produto $p$ no nó $i\left(\forall i \in H t, p \in P_{i}\right)$.

\section{Constantes:}

$J_{i}: 1$ se $i \in H p,-1$ se $i \in H t$.

\section{Variáveis de Decisão:}

$x_{\text {imjnv }}: 1$ se o $\operatorname{arco}(i, m, j, n)$ é percorrido pelo navio $v, 0$ caso contrário;

$z_{\text {imv }}: 1$ se o navio $v$ termina sua rota na visita $m$ do PO $i, 0$ caso contrário;

$y_{\text {im }}: 1$ se a visita $m$ ao PO $i$ não ocorre, 0 caso contrário;

$\sigma_{i m \tau}: 0$ se a visita $m$ ao PO $i$ se sobrepõe a visita anterior $\tau$, 1 caso contrário.

$l_{\text {impv }}$ : carga de produto $\mathrm{p}$ a bordo do navio $v$ depois da visita $m$ ao PO $i$, em $\mathrm{m}^{3}$;

$q_{\text {impv }}$ : quantidade carregada ou descarregada de produto $p$ pelo do navio $v$ após a visita $m$ ao PO $i$, em $\mathrm{m}^{3}$. 
$t s_{i m}$ : instante de início da visita $m$ do PO $i$ em horas;

$t e_{i m}$ : instante de término da visita $m$ do PO $i$ em horas.

$s s_{i m p}$ : estoque do produto $p$ no PO $i$ no instante de inicio da visita $m, \mathrm{em} \mathrm{m}^{3}$;

$s e_{i m p}$ : estoque do produto $p$ no PO $i$ no instante de término da visita $m$, em $\mathrm{m}^{3}$;

$s f_{i p}$ : estoque do produto $p$ no $\mathrm{PO} i$ no final do horizonte de planejamento, $\mathrm{em}^{3}$.

Considera-se que algumas variáveis são previamente fixadas, por exemplo: $l_{i_{v} 1 p v}=$ $l o_{p v}, t s_{i_{v} 1}=$ liberacao $_{v}, t e_{i_{v} 1}=$ liberacao $_{v}$, dentre outras. Também impõe-se que $t s_{i m} \leq T$.

$\left.\operatorname{Minimizar}\left(f=\sum_{i \in H_{v}} \sum_{m \in M_{i}} \sum_{j \in H_{v} \backslash\left\{i_{v}\right\}} \sum_{n \in M_{j}} \sum_{v \in V} x_{i m j n v}\left(t_{i j}\left(c m_{v}-c s b_{v}\right)\right)+c a_{j}\right)\right)$

Sujeito às restrições:

$$
\begin{aligned}
& \sum_{j \in H_{v} \backslash\left\{i_{v}\right\}} \sum_{n \in M_{j}} x_{i_{v} 1 j n v}+z_{i_{v} m v}=1,(\forall v \in V) \\
& \sum_{j \in H_{v}} \sum_{n \in M_{j}} x_{j n i m v}=\left(\sum_{j \in H_{v} \backslash\left\{i_{v}\right\}} \sum_{n \in M_{i v}} x_{i m j n v}\right)+z_{i m v},\left(\forall v \in V, i \in H_{v} \backslash\left\{i_{v}\right\}, m \in M_{i}, v \in V\right) \\
& \sum_{j \in H_{v}} \sum_{n \in M_{i}} \sum_{v \in V} x_{\text {jnimv }}=1-y_{i m},\left(\forall i \in H_{v} \backslash\left\{i_{v}\right\}, m \in M_{i}\right) \\
& y_{i m}=y_{i(m-1)} \geq 0,\left(\forall i \in H_{v} \backslash\left\{i_{v}\right\}, m \in M_{i}, m \neq 1\right) \\
& l_{\text {jnpv }} \geq l_{\text {impv }}+J_{j} q_{j n p v}-C A P_{v}\left(1-x_{i m j n v}\right), \quad\left(\forall v \in V, i \in H_{v}, m \in M_{i}, j \in H_{v} \backslash\left\{i_{v}\right\}, n \in M_{j}\right) \\
& l_{\text {jnpv }} \leq l_{\text {impv }}+J_{j} q_{j n p v}+C A P_{v}\left(1-x_{i m j n v}\right), \quad\left(\forall v \in V, i \in H_{v}, m \in M_{i}, j \in H_{v} \backslash\left\{i_{v}\right\}, n \in M_{j}\right) \\
& \sum_{j \in H_{v}} \sum_{n \in M_{j}} x_{\text {jnimv }} \geq \frac{\sum_{p \in P_{i p}} q_{i m p v}}{C A P_{v}},\left(\forall v \in V, i \in H_{v} \backslash\left\{i_{v}\right\}, m \in M_{i}\right) \\
& \sum_{p \in P T} l_{i m p v} \leq C A P_{v} \sum_{j \in H_{v}} \sum_{n \in M_{j}} x_{\text {jnimv }},\left(\forall v \in V, i \in H_{v} \backslash\left\{i_{v}\right\}, m \in M_{i}\right) \\
& \sum_{p \in P_{i}} \sum_{v \in V} q_{i m p v} \geq Q M I N_{i m}\left(1-y_{i m}\right),\left(\forall i \in H_{v} \backslash\left\{i_{v}\right\}, m \in M_{i}\right) \\
& \sum_{m \in M_{i}} \sum_{v \in V} q_{i m p v}=\operatorname{dem}_{i p},\left(\forall i \in H t \cap H_{v}, p \in P_{i}\right) \\
& \sum_{p \in P T} l_{j n p v} \leq \alpha_{j v} C A P_{v}+\sum_{p \in P_{j}} q_{j n p v}+\left(1-\sum_{i \in H_{v}} \sum_{m \in M_{i}} x_{i m j n v}\right) C A P_{v}, \\
& \left(\forall v \in V, j \in\left(H t \cap H_{v}\right), n \in M_{j},(i, m, j, n, v) \in A\right) \\
& \sum_{p \in P T} l_{j n p v} \leq 0,5 C A P_{v}+\sum_{p \in P_{j}} q_{j n p v}+(1-0,5)\left(1-\sum_{i \in H_{v}} \sum_{m \in M_{i}} x_{i m j n v}\right) C A P_{v}, \\
& \left(\forall v \in V D P, j \in\left(H p \cap H_{v}\right), n \in M_{j}\right) \\
& \sum_{p \in P T} l_{j n p v} \leq 0,3 C A P_{v}+\sum_{p \in P_{j}} q_{j n p v}+(1-0,3)\left(1-\sum_{i \in H_{v}} \sum_{m \in M_{i}} x_{i m j n v}\right) C A P_{v}, \\
& \left(\forall v \in V, v \notin V D P, j \in H p, j \notin P F, n \in M_{j}\right) \\
& \sum_{i \in H_{v}} \sum_{m \in M_{i}} x_{i m j n v}=0\left(\forall v \in V, v \notin V D P, j \in P F, n \in M_{j}\right) \\
& t e_{i m}=t s_{i m}+T S \sum_{j \in H_{v}} \sum_{n \in M_{j}} \sum_{v \in V} x_{j n i m v},\left(\forall i \in H_{v} \backslash\left\{i_{v}\right\}, m \in M_{i},(i, m, j, n, v) \in A\right) \\
& t s_{j n} \geq t e_{i m}+t_{i j}-2 T\left(1-x_{i m j n v}\right), \quad\left(\forall i \in H_{v}, m \in M_{i}, j \in H_{v} \backslash\left\{i_{v}\right\}, n \in M_{j}, v \in V\right) \\
& t s_{i m} \geq t e_{i(m-1)},\left(\forall i \in H p, m \in M_{i}, m \neq 1\right) \\
& t s_{i m} \geq t s_{i(m-1)},\left(\forall i \in H t, m \in M_{i}, m \neq 1\right) \\
& s s_{i 1 p}=s o_{i p}+R_{i p} t s_{i 1},\left(\forall i \in H p, p \in P_{i}\right) \\
& s e_{i m p}=s s_{i m p}-\sum_{v \in V} q_{i m p v}+R_{i p}\left(t e_{i m}-t s_{i m}\right),\left(\forall i \in\left(H p \cap H_{v}\right), m \in M_{i}, p \in P_{i}\right) \\
& s s_{i m p}=s e_{i(m-1) p}+R_{i p}\left(t s_{i m}-t e_{i(m-1)}\right),\left(\forall i \in H p, m \in M_{i}, p \in P_{i}, m \neq 1\right) \\
& \text { SMin }_{i p} \leq s s_{i m p} \leq \operatorname{SMax}_{i p},\left(\forall i \in H p, m \in M_{i}, p \in P_{i}\right) \\
& \text { SMin }_{i p} \leq \operatorname{se}_{i m p} \leq \operatorname{SMax}_{i p},\left(\forall i \in H p, m \in M_{i}, p \in P_{i}\right)
\end{aligned}
$$




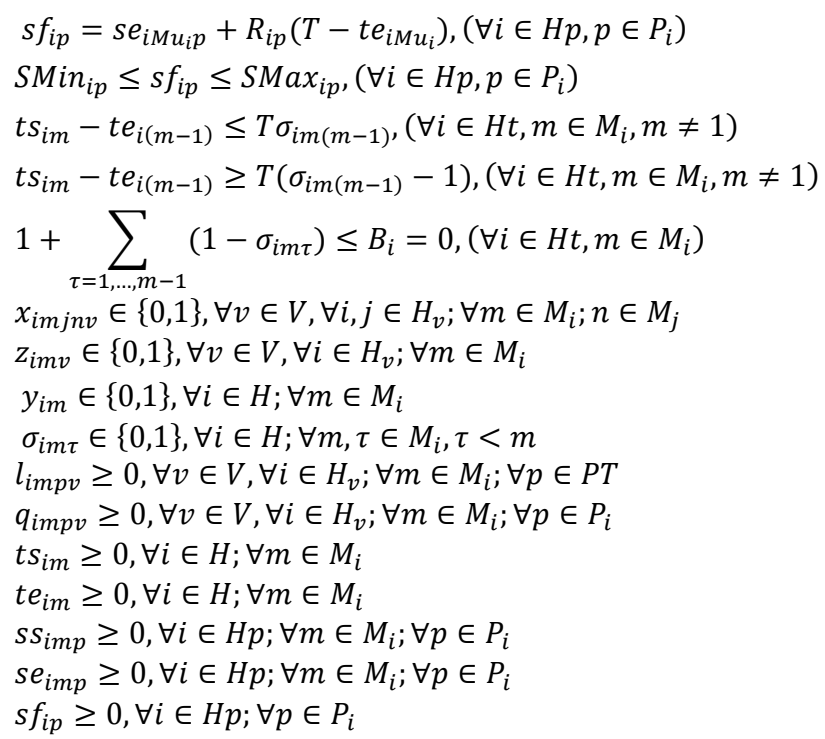

O objetivo do modelo (1) é o de minimizar o custo de movimentação dos navios e o custo de atracação em cada $\mathrm{PO}$ visitado. O custo de stand by (custo do navio parado) faz parte do custo fixo de cada navio e este custo é descontado do custo de movimento nos instantes de deslocamento do navio.

As restrições de fluxo de rede, (2) e (3), garantem que os navios terminem sua rota na sua posição atual ou partem para algum ponto operacional da rede. As restrições (4) indicam quais vistas ocorreram ou não a cada ponto e as restrições (5) ordenam as visitas realizadas.

As restrições de carregamento e descarregamento de navios, (6) e (7), controlam a carga a bordo do navio após cada visita realizada. Elas são resultado de uma linearização na restrição $x_{i m j n v}\left(l_{i m p v}+J_{j} q_{j n p v}-l_{j n p v}\right)=0$, que indica que se a visita ocorre a carga a bordo do navio é igual à carga no início da visita, mais (menos) a quantidade carregada (descarregada). As restrições (8) garantem que toda visita a um ponto operacional seja acompanhada de um serviço (coleta ou entrega). As restrições (9) garantem que a carga a bordo do navio não exceda sua capacidade e as restrições (10) por sua vez limitam o carregamento ou descarregamento a um lote mínimo.

As restrições (11) garantem que a demanda de cada terminal seja completamente atendida durante todo o horizonte de planejamento.

As restrições de atracação relacionadas ao calado máximo dos navios (12) permitem a atracação de determinados navios em alguns terminais desde que sua capacidade ocupada seja menor do que $80 \%$ de sua capacidade total. Para plataformas, as restrições de atracação (13) a (15), foram formuladas respeitando as condições descritas na Tabela 1, impedindo atracação de navios com DP em plataformas fixas e permitindo as demais atracações com navios com capacidade ocupada limitada a uma porcentagem da capacidade total.

As restrições temporais, (16) a (19), descrevem o tempo de início e término das visitas realizadas, sendo que as restrições (17) são resultado de uma linearização da restrição $x_{i m j n v}\left(t e_{i m}+t_{i j}-t s_{j n}\right)=0$, que relaciona os instantes de término e início de duas visitas consecutivas de um mesmo navio. As restrições de estoque nas plataformas, (20) a (26) calculam o estoque durante a visita (21), no início de cada visita (20 e 22) e no final do horizonte de planejamento (25) para cada plataforma e garantem que os níveis de estoque em cada ponto estejam entre os limitantes máximos e mínimos de estocagem. (23, 24 e 26).

As restrições (27) e (28) contabilizam quantos navios estão atracados ao mesmo tempo em cada terminal e as restrições (29) garantem que essa quantidade não exceda o número de berços do terminal.

Finalmente, as restrições (30) a (40) determinam o domínio das variáveis.

O modelo (1)-(40) foi implementado na linguagem de modelagem GAMS 24.0.1, e exemplares com dados reais da operação da empresa petrolífera estudada foram resolvidos utilizando-se o sofware de otimização CPLEX 12.5.0.1. 


\subsection{UM MÉTODO DE SOLUÇÃO HEURÍSTICO}

Com base nas características do problema real da empresa do presente estudo de caso, foi desenvolvido um método heurístico com procedimento de eliminação de operações de coleta motivada apenas para aliviar estoques das plataformas (operações de alívio) e uma busca local de troca de navios. Os passos da heurística, do procedimento de eliminação de alívios (EA) e da busca local (BL) são descritos nos Quadros 1, 2 e 3, respectivamente.

\section{Dados de entrada adicionais:}

$C A P d_{v 0}$ : capacidade de carga disponível no navio $v$ no início do horizonte de planejamento (CAP: ); liberacao $_{v 0}$ : instante atual de liberação do navio $v$;

posicao $_{v 0}$ : posicao atual do navio $v$;

$s_{p 0}$ : nível de estoque de produto $p$ (na plataforma $i_{p}$ ) no início do horizonte de planejamento ( $s o_{i p}$ ); dterminal $_{t p 0}$ : demanda não satifsfeita do produto $p$ pelo terminal $t$ no início do horizonte de planejamento $\left(\right.$ dem $\left._{i p}\right)$.

$M$ : valor de inicialização da solução incumbente.

\section{Variáveis adicionais:}

$t$ : tempo decorrido, em segundos;

$s_{i_{p} m_{i_{p}}}$ : nível de estoque de produto $p$ na plataforma $i_{p}$ após sua $m$ - ésima visita;

liberacao $_{v}$ : instante atual de liberação do navio $v$;

posicao $_{v}$ : posicao atual do navio $v$;

$C A P d_{v}$ : capacidade de carga disponível no navio $v$;

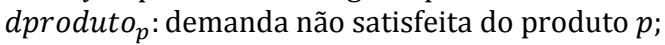

dterminal $_{\text {tpm }_{t}}$ : demanda não satifsfeita do produto $p$ pelo terminal $t$ após sua $\mathrm{m}$ - ésima visita;

dtotal: demanda total atual de produtos;

top $p_{i_{p} m_{i_{p}}}$ : instante em que a plataforma $i_{p}$ atinge o estoque máximo após sua $\mathrm{m}$ - ésima visita;

$q_{i p m}$ : quantidade coletada ou descarregada do produto $p$ no ponto $i$ em sua $\mathrm{m}-$ ésima visita . 
Quadro 1 - Passos da Heurística Construtiva.

1. Inicialização das variáveis;

2. Escolha a próxima plataforma a ser visitada $i_{p}{ }^{*}$ (e, portanto, o próximo produto $p^{*}$ a ser coletado) pela seleção aleatória de uma das seguintes regras: (RP1) menor $t o p_{i_{p} m_{i_{p}}}$, (RP2) maior taxa de produção $R_{i_{p}}$, (RP3) maior demanda, (RP4) plataformas fixas de menor top $_{i_{p} m_{i_{p}}}$.

3. Se a demanda por produto $p^{*}$ for nula vá para o passo 4 (operação de alívio); caso contrário, vá para o passo 5 (operação de atendimento de demanda).

4. Se o estoque máximo da plataforma $i_{p}{ }^{*}$ for atingido após o final do horizonte de planejamento, siga para o passo 4.1, caso contrário vá para o passo 4.2.

4.1. Penalize a plataforma $i_{p}{ }^{*}$ para que não seja escolhida novamente. Se ainda houver demanda por outros produtos, volte para o passo 2 e selecione a próxima plataforma a ser visitada; caso contrário, finalize o procedimento.

4.2 Identifique os navios capazes de realizar a coleta em $i_{p}{ }^{*}$ até o instante $t o p_{i_{p}}{ }^{*} m_{i_{p}}{ }^{*}$.

4.2.1 Designe o navio $v^{*}$ para realizar a coleta em $i_{p}{ }^{*}$ pela escolha aleatória de uma das seguintes regras: (RV1) menor custo; (RV2) menor custo por unidade de capacidade; (RV3) maior capacidade; (RV4) menor distância de $i_{p}{ }^{*}$; (RV5) compatível a menos pontos operacionais, em caso de empate, menor custo;

4.2.2 Calcule as quantidades coletadas nas plataformas e entregues nos terminais visitados e atualize o estoque das plataformas visitadas e a posição, o instante de liberação e a capacidade de $v^{*}$, a demanda total, a demanda dos produtos entregues e a demanda de produtos entregues por terminal visitado, adicione à função objetivo $f$ os custos de movimentação e atracação realizados e retorne ao passo 2.

5. Selecione o terminal $t^{*}$ que demanda o produto $p^{*}$ por uma das seguintes regras: (RT1) maior demanda por $p^{*}$, (RT2) menor demanda por $p^{*}$, (RT3) menor distância de $i_{p}{ }^{*}$, (RT4) maior distância de $i_{p}{ }^{*}$.

5.1 Identifique os navios capazes de realizar a coleta do produto $p^{*}$ em $i_{p}{ }^{*}$ até o instante top $_{i_{p^{*}} m_{i_{p^{*}}}}$ e a entrega em $t^{*}$ antes do final do horizonte de planejamento.

5.2 Designe o navio $v^{*}$ para realizar a coleta em $i_{p}{ }^{*}$ e a entrega em $t^{*}$ por uma das regras RV1, RV2, RV3, RV4, RV5 (descritas no passo 2) e (RV6) menor custo que comporte a demanda de $i_{p}{ }^{*}$.

5.3 Se não houver capacidade excedente no navio $v^{*}$ após coletar a demanda de produto $p^{*}$ do terminal $t^{*}$ suficiente para coletar outra demanda não atendida, vá para o passo 4.2.2; caso contrário, vá para o passo 5.3.2.

5.3.2 Das demandas por um mesmo produto $p^{*}$, ou de um mesmo terminal $t^{*}$, selecione aquelas que podem ser totalmente coletadas pelo navio $v^{*}$;

5.3.2.1 Se nenhuma das possíveis rotas obtidas no passo anterior for factível, vá para o passo 5.3.1 Caso contrário:

5.3.2.2 Escolha a rota factível que visita mais pontos operacionais, e em caso de empates, a de menor custo e volte ao passo 4.2.2.

\section{Quadro 2 - Passos do Processo de Eliminação de Alívios (EA).}

1. A partir da solução gerada pela heurística construtiva, identifique todas as plataformas $i_{p}$ que segundo o plano necessitaram de alívio em alguma visita $m_{i_{p}}$. Se não houver operações de alívio, finalize o procedimento. Caso contrário, selecione a plataforma $i_{p}{ }^{*}$ c ommenor quantidade coletada $q_{i_{p}{ }^{*}, p^{*}, m_{i_{p}}{ }^{*}}$.

2. Identifique os navios $v$ que realizaram as demais vistas (operação de atendimento de demanda) à plataforma $i_{p}{ }^{*}$;

3. Para cada navio $v$ que visita a plataforma $i_{p}{ }^{*}$, identifique os navios $w$ compatíveis com $v$, ou seja, que tenham as seguintes características: (i) não foi utilizado no roteamento; (ii) seja compatível com todos os pontos da rota de $v$ e; (iii) $C A P d_{w} \geq$

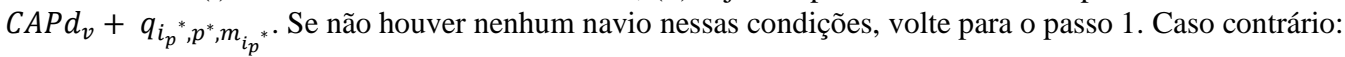

4. Calcule as economias de trocar cada navio $v$ por cada navio compatível $w$. Se não houver nenhuma troca que implique em uma economia positiva volte para o passo 1 . Caso contrário:

5.Selecione o navio $v^{*}$ e o navio e $w^{*}$ que representem a troca com maior economia, e realize a troca.

6. Retire a $m_{i_{p}}{ }^{*}$-ésima visita a plataforma $i_{p}{ }^{*}$ do roteamento, diminua todas as visitas posteriores a $m_{i_{p}}{ }^{*}$ a plataforma $i_{p}{ }^{*}$ em uma unidade e volte ao passo 1. 
1. Parta de uma solução inicial (determinada pela heurística construtiva ou pela heurística construtiva+EA). Para cada navio $v$ utilizado na solução inicial identifique os navios $w$ (utilizado ou não no roteamento) compatíveis com $v$, que sejam capazes de realizar a mesma rota e manter as quantidades entregues da rota de $v$, tendo as seguintes características: (i) Compatibilidade do navio $w$ com os pontos da rota do navio $v$; (ii) Atendimento das restrições temporais $\left(t s_{i m} \leq t o p_{i_{p} m_{i_{p}}}\right.$ );

(iii) Capacidade suficiente para entregar as mesmas quantidades;

2. Calcule as economias de trocar cada navio $v$ utilizado por um navio compatível $w$. Se não houver nenhuma troca compatível, vá para o passo 4. Caso contrário,

3. Selecione o navio $v^{*}$ e o navio e $w^{*}$ que representem a troca com maior economia, realize a troca de $v^{*}$ por $w^{*}$, calcule as quantidades coletadas por $w^{*}$, considere o navio $v^{*}$ como não utilizado, o navio $w^{*}$ incompatível com todos os navios utilizados e volte ao passo 2 ;

4. Se não existir nenhuma plataforma que necessite de um novo alívio siga para o passo 6. Caso contrário identifique cada plataforma $i_{p}{ }^{*}$ que necessita de novo alívio, e calcule os custos viagem de cada navio $w$, compatível com esta plataforma, para realizar a nova visita a mesma.

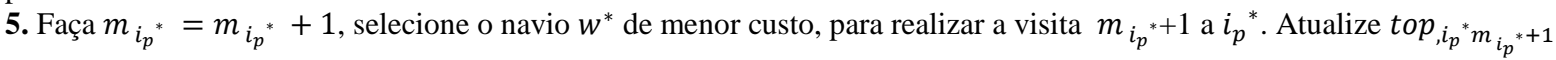
e volte ao passo 4 .

6. Se o custo total após as trocas do passo 3 e novos alívios, realizados no passo 4 for maior que o custo da solução inicial, finalize o procedimento. Caso contrário, realize as trocas e os alívios e finalize o procedimento.

$\mathrm{O}$ método heurístico descrito acima foi implementado em linguagem $\mathrm{C}$ utilizando o pacote de programas Visual Studio 2010. O procedimento descrito no Quadro 1 foi executado por Tmax segundos para gerar diferentes soluções. O procedimento de eliminação de alívios (EA), descrito no Quadro 2, e o de busca local (BL), descrito no Quadro 3, foram então aplicados à melhor solução encontrada. O Quadro 4 resume os passos do método heurístico completo.

Quadro 4 - Passos do Método Heurístico.

1. Faça $f^{*}=\infty, S^{*}=\emptyset$.

2. Enquanto $t \leq T \max$, repita:

2.1 Aplique a heurística construtiva descrita no Quadro 1, obtendo a solução $S$ com valor da função objetivo $f$.

2.2 Se $f<f^{*}$, faça $f^{*}=f$ e $S^{*}=S$.

3. Aplique EA (descrito no Quadro 2) seguido de BL (descrito no Quadro 3) à S*, e retorne a solução obtida.

\section{EXPERIMENTOS COMPUTACIONAIS}

\subsection{DETALHAMENTO DAS INSTÂNCIAS}

Os dados das operações reais de coleta e entrega fornecidos pela empresa consideram horizontes de planejamento em uma escala de tempo horária e incluem as informações sobre (i) a frota (número de navios, instante e posição de liberação, custos, capacidade, velocidade, existência ou não de sistema de posicionamento dinâmico, entre outros), (ii) os pontos operacionais (número, distância entre cada par), (iii) plataformas (tipo, produto extraído, taxa de produção, estoque máximo, estoque mínimo, entre outros), e (iv) os terminais (quantidade de berços, loa e calado máximo permitido).

Os dados fornecidos pela empresa estudada são referentes a um mês de operação (Janeiro/2013), totalizando 83 pedidos pré-definidos de produtos. Quatro instâncias, foram geradas selecionando alguns pedidos em ordem cronológica cujos nomes refletem a quantidade de pedidos selecionados, por exemplo, a instância intitulada "N10" é composta pelos 10 primeiros pedidos de produtos do conjunto total de dados. A Tabela 2, a seguir, caracteriza as instâncias em relação a número de pontos operacionais (plataformas e terminais), navios e tamanho do horizonte de planejamento em horas. 
Tabela 2 - Características das instâncias.

\begin{tabular}{|c|c|c|c|c|}
\hline Instância & Plataformas/Produtos & Terminais & Navios & Horizonte de Planejamento (h) \\
\hline N6 & 4 & 1 & 25 & 197 \\
\hline N10 & 6 & 3 & 25 & 243 \\
\hline N12 & 8 & 3 & 25 & 267 \\
\hline N16 & 9 & 4 & 25 & 291 \\
\hline
\end{tabular}

Informações sobre os lotes de produtos em cada pedido foram utilizadas de forma a calcular a demanda total de produtos em cada terminal, a qual deve ser entregue durante o horizonte de planejamento em uma ou mais visitas. Especificamente, para cada produto $p$ solicitado por um terminal $i$ no horizonte de planejamento em questão, foram somados os tamanhos dos lotes de $p$ de todos os pedidos. Esta soma corresponde à demanda de $i$ por $p$ $\left(\right.$ dem $\left._{i p}\right)$, utilizada na restrição 11 do modelo (1)-(40) descrito na Seção 3.2.

\subsection{ANÁLISE DOS RESULTADOS}

O modelo matemático, descrito na seção 3.2, foi implementado na linguagem de modelagem GAMS 24.0.1 e resolvido utilizando o sofware de otimização CPLEX 12.5.0.1, com as opções de ênfase em encontrar uma solução factível com um bom valor da função objetivo ( mipemphasis $=4$, fpheur $=2$ ), os critérios de parada são gap nulo de otimalidade ou 18000 segundos (5 horas) de processamento (tilim $=18000)$.

O método heurístico, descrito na seção 3.3, foi implementado em linguagem C e resolvido utilizando o pacote de programas Visual Studio 2010. Para cada instância descrita na Tabela 2, a fase de construção de soluções (Quadro 1), gerou um conjunto de soluções factíveis, a partir da escolha aleatória de regras fixas de escolha de plataformas, terminais e navios, até atingir o critério de parada de $\operatorname{Tmax}=100$ segundos de processamento. Conforme discutido na Seção 3.3, a melhor solução gerada na fase de construção passa por dois procedimentos de melhoria, EA e BL, descritos nos Quadros 2 e 3, respectivamente. O tempo total de execução de cada instância permaneceu em 100 segundos uma vez que os procedimentos de melhoria eram executados apenas para a melhor solução e a duração da execução é menor que 1 segundo. A Tabela 3 apresenta os resultados com o método exato e com o método heurístico.

Tabela 3 - Resultados Computacionais.

\begin{tabular}{|c|c|c|c|}
\hline N6 & $\boldsymbol{f}$ & Gap (\%) & Tempo (s) \\
\hline Modelo Matemático & 1022.9 & 0 & 710 \\
\hline Método Heurístico & 1079.2 & 5.2 & 100 \\
\hline N10 & $\boldsymbol{f}$ & Gap (\%) & Tempo (s) \\
\hline Modelo Matemático & 1455.3 & 0 & 5670 \\
\hline Método Heurístico & 1462.9 & 0.5 & 100 \\
\hline N12 & $\boldsymbol{f}$ & Gap (\%) & Tempo (s) \\
\hline Modelo Matemático & 2002.7 & 31.2 & 18000 \\
\hline Método Heurístico & 1897.7 & 26.1 & 100 \\
\hline N16 & $\boldsymbol{f}$ & Gap (\%) & Tempo (s) \\
\hline Modelo Matemático & - & - & 18000 \\
\hline Método Heurístico & 2424.8 & 22.8 & 100 \\
\hline Modelo Matemático (S0) & 2303.7 & 17.8 & 18000 \\
\hline
\end{tabular}

A resolução do modelo matemático forneceu a solução ótima para as instâncias N6 e N10. Já para instância N12 encontrou-se uma solução com um gap de 30\%. Dado que o CPLEX não determinou solução factível para a instância N16 após 5 horas de processamento, a solução obtida pelo método heurístico para a instância N16, foi utilizada como solução de partida (S0) para o modelo para determinar o gap e verificar se o CPLEX melhoraria essa solução após mais 5 horas de processamento.

O método heurístico gerou soluções com custos até 5.2\% acima do custo da solução obtida pelo CPLEX para as instâncias N6, N10 e N16, enquanto uma solução com custos 5.5\% abaixo dos custos da solução encontrada pelo CPLEX foi obtida para a instância N12. 
Foram realizados ainda testes com a heurística com todas as diferentes combinações de regras, 120 combinações no total, para verificar quais seriam as melhores regras de escolha de plataformas, navios para operação de alívio e navios para operação de coleta e entrega, para cada uma das quatro instâncias. As regras de escolha de terminais (RT1, RT2, RT3 ou RT4, descritas no passo 5 da heurística detalhada no Quadro1), não puderam ser analisadas a partir das instâncias testadas, dado que em todas elas cada plataforma atendia demanda de um único terminal.

Após as execuções das diferentes combinações de regras constatou-se que, para o conjunto de dados analisado, as regras que merecem destaque pela qualidade relativa das soluções obtidas são: para escolha de plataformas, RP2 (maior taxa de produção) e RP3 (maior demanda), para escolha de navios para operação de alívio, RV1 (menor custo) e para a escolha de navios para operação de coleta e entrega, RV5 (compatível com menor número de pontos operacionais e em caso de empate, de menor custo). A Tabela 4 aponta os resultados para as 4 instâncias analisadas com regras fixas para todas as iterações a partir de duas combinações diferentes: RP2, RV1 e RV5; e RP3, RV1 e RV5. Os procedimentos de melhoria EA e BL foram executados nas soluções.

Tabela 4 - Melhores Regras Fixas.

\begin{tabular}{|c|c|c|c|}
\hline N6 & $\boldsymbol{f}$ & Gap (\%) & Tempo (s) \\
\hline RP2;RV1;RV5 & 1158.1 & 11.6 & 1 \\
\hline RP3;RV1;RV5 & 1158.1 & 11.6 & 1 \\
\hline N10 & $\boldsymbol{f}$ & Gap (\%) & Tempo (s) \\
\hline RP2;RV1;RV5 & 1501.5 & 3.1 & 1 \\
\hline RP3;RV1;RV5 & 1480.0 & 1.6 & 1 \\
\hline N12 & $\boldsymbol{f}$ & Gap (\%) & Tempo (s) \\
\hline RP2;RV1;RV5 & 1988.8 & 29.5 & 2 \\
\hline RP3;RV1;RV5 & 1945.5 & 27.9 & 2 \\
\hline N16 & $\boldsymbol{f}$ & Gap (\%) & Tempo (s) \\
\hline RP2;RV1;RV5 & 2509.5 & 24.5 & 2 \\
\hline RP3;RV1;RV5 & 2548.1 & 25.7 & 2 \\
\hline
\end{tabular}

Visando a melhoria da qualidade das soluções obtidas pelo método heurístico de busca local, descrito no Quadro 4, pode-se priorizar a escolha de regras que determinem melhores resultados, como as apontadas na Tabela 4 , e em contrapartida, diminuir a frequência de escolha das regras que resultem em soluções de menor qualidade.

\section{CONCLUSÕES E TRABALHOS FUTUROS}

O presente artigo apresentou um problema de programação e roteirização de navios com controle de estoque nos pontos de suprimento, encontrado nas operações reais de coleta e entrega de diferentes tipos de óleos cru de uma empresa petrolífera nacional. Exemplares com dados reais foram resolvidos por meio de uma implementação da modelagem matemática e de um método heurístico de busca local. O método heurístico determinou soluções factíveis de qualidade para instâncias de pequeno porte (com até 5.2\% de gap) e para instâncias de maior porte para as quais o modelo matemático resolvido pelo software CPLEX não encontrou soluções, em um tempo de processamento de 1 a 2 segundos por instância testada. Para a geração de soluções para exemplares de maior porte (com horizonte de planejamento de até 720 horas), cujos dados já foram fornecidos pela empresa estudada, almeja-se investir em diferentes procedimentos de busca local, como troca de sub rotas (sub rotas geradas a cada iteração) entre navios, troca da ordem das sub rotas de cada navio, respeitando as restrições temporais, entre outras. A possibilidade da implementação de meta-heurísticas, como o Simulated Annealing (Kirkpatrick et al.,1983) e o algoritmo Eletromagnético (Birbil and Fang, 2003) será estudada nas próximas etapas da presente pesquisa. 


\section{REFERÊNCIAS}

[1] Agra, A., Christiansen, M., \& Delgado, A. Mixed Integer Formulations for a Short Sea Fuel Oil Distribution Problem. Transportation Science, Vol. 47, No 1, pp. 108-124, February 2013.

[2] Agra, A., Christiansen, M., Delgado, A., Simonetti, L. Hybrid heuristics for a short sea inventory routing problem. European Journal of Operational Research 236 pp. 924-935, 2014.

[3] Al-Khayyal, F., Hwang, S. J. Inventory Constrained Maritime Routing and Scheduling for Multi-Commodity Liquid Bulk, Part I: Applications and model. European Journal of Operational Research 176 pp. 106-130, 2007.

[4] Birbil, S. I., Fang, S. C. An Electromagnetism-like Mechanism for Global Optimization. Journal of Global Optimization 25: 263-282, 2003.

[5] Christiansen, M., Nygreen, A, Modeling path flows for a combined ship routing and inventory management problem, Annals of Operations Research 82 pp.391-421, 1998a.

[6] Christiansen, M., Nygreen, A. A method for solving ship routing problems with inventory constraints, Annals of Operations Research 81 pp. 357-378, 1998b.

[7] Christiansen, M., Decomposition of a Combined Inventory and Time Constrained Ship Routing Problem. Trasportation Science, vol. 33, no. 1, pp. 3-16, 1999.

[8] Christiansen, M., Fagerholt, K., Flatberg, T., Haugen, O., Kloster, O., Lund, E. H. Maritime inventory routing with multiple products: a case of study from the cement industry. Europen Journal of Operational Research 208 (1), 86-94, 2011.

[9] Christiansen, M., Fagerholt, K., Nygreen, B., Ronen, D. Ship routing and scheduling in the new millennium. European Journal of Operational Research, 228 pp.467-483,_2013.

[10] Fagerholt, K. Optimal fleet design in a ship routing problem. International Transactions in Operations Research 6 pp. 453-464, 1999.

[11] Halvorsen-Weare, E. E., Fagerholt, K. Routing and scheduling in a liquefied natural gas shipping problem with inventory and berth constraints. Annals of operational research 203 pp.167-186, 2010.

[12] Hwang, S. J. Inventory Constrained Maritime Routing and Scheduling for MultiCommodity Liquid Bulk. School of Industrial and Systems Engineering Georgia Institute of Technology. May 2005.

[13] Kirkpatrick, S, Gelatt, C.D., Vecchi, M.P. 1983. Optimization by Simulated Annealing. Science, vol 220, No. 4598, pp671-680, 1983.

[14] Li, J., Karimi, I. A., Srinivasan, R. Efficient bulk maritime logistics for the supply and delivery of multiple chemicals. Computer \& Chemical Engineering 34 (12), pp. 21182128, 2010.

[15] Miller, M. An interactive, computer-aided ship scheduling system, European Journal of Operational Research 32 pp. 363-379, 1987.

[16] Persson, J. A., Göthe-Lundgren, M. Shipment planning at oil refineries using column generation and valid inequalities. European Journal Operational Research 163, pp. 631652, 2005.

[17] Revista Notícias do Trecho. Plataforma Fixa e FPSO. Disponível em: <http://www.noticiasdotrecho.com.br/2014/06/ultimas-noticias-petrobras-estuda.html>. Acesso em 05/12/2014.

[18] Siswanto, K., Essam, D., Sarker, R. - Solving the ship inventory routing and scheduling problem with undedicated compartments. Computers and Industrial Engineering 61 (2) pp. 289-299, 2011.

[19] SOBERANA - Sociedade Brasileira de Engenharia Naval - Principais medidas dimensões e características do navio. Disponível em: <http://www.soberana.org.br/downloads/diciona_naval/Principais\%20Medidas.pdf>. Acesso em: 01/03/2015.

\section{Agradecimentos}

À CAPES pelo financiamento da pesquisa. 